\title{
Análisis de la participación ciudadana en el otorgamiento de permisos de aprovechamiento forestal en el chocó $2009-2013^{*}$
}

\author{
Analysis of public participation in the permit granting process for the use of forestry in Chocó 2009-2013
}

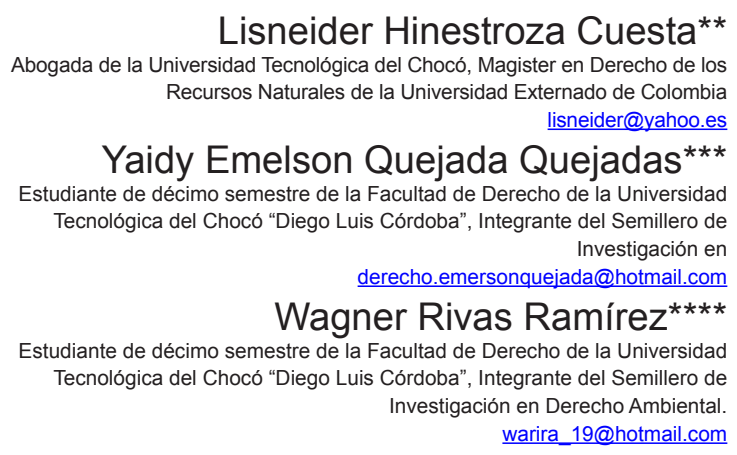

\section{Resumen}

Este trabajo tiene como objetivo analizar los resultados obtenidos sobre la participación ciudadana en el proceso de otorgamiento de permisos de aprovechamiento forestal otorgados por la Corporación Autónoma Regional para el Desarrollo Sostenible del Departamento del Chocó (CODECHOCÓ) en el periodo comprendido entre 2009-2013. Para la ejecución de este proyecto se utilizó el método de Investigación Descriptivo propuesto por (Toro, \& Parra, 2010, p. 244) el cual pretende dar cuenta de la realidad; se interpuso derecho de petición a la autoridad ambiental, dan cuenta del otorgamiento de 224 autorizaciones de aprovechamiento forestal persistente y 12 autorizaciones de aprovechamiento forestal único. Se seleccionó al azar una muestra representativa de 24 expedientes, que corresponden al $10 \%$ de los permisos otorgados. Se concluye que en el otorgamiento de autorizaciones de aprovechamiento forestal, la participación de la comunidad en el departamento del Chocó, es esporádica.

Palabras clave: Aprovechamiento forestal, autorizaciones de aprovechamiento forestal, participación ciudadana, actuación administrativa, autoridad ambiental.

\begin{abstract}
This paper aims to analyze a study carried out on public participation in the process of granting logging permits by the Regional Autonomous Corporation for the Sustainable Development of the Province of Chocó ("CODECHOCÓ”) during 2009 to 2013. To conduct this research project, a descriptive research method was used (Toro \& Parra, 2010, p. 244). This research method seeks to explain the reality being researched. A right of petition was filed with the environmental authority CODECHOCO. As a result of this, information was received indicating that 224 persistent forestry permits were granted, in addition to 12 exclusive logging requests. A representative sample of 24 files (10\% of the permits granted) were selected for the purpose of this project. We conclude that in granting of permits for the use of environmental resources which directly impact the environment, the participation of the community in the province of Choco is sporadic.
\end{abstract}

Keywords: Logging permits, authorization for the use of forestry, public participation, administrative action, Environmental authority.

" El artículo que se presenta a continuación es un artículo de revisión producto del proyecto de Investigación: "Formación en mecanismos de participación ciudadana para la intervención en actuaciones administrativas de otorgamiento de permisos ambientales en el departamento del Chocó 2009-2013". Proyecto financiado por la Universidad Tecnológica de Chocó “Diego Luis Córdoba” y el Departamento Administrativo de Ciencia, Tecnología e Innovación COLCIENCIAS. Convocatoria Colciencias 617 de 2013 


\section{Introducción}

A partir de la expedición de la Constitución Política de Colombia de 1991, en nuestro país, tal como lo explica Amaya (2010, p.133) se acoge la protección y defensa del medio ambiente como una obligación en cabeza del Estado y de los particulares, como un deber y un derecho colectivo. De ahí que, la Carta Constitucional consagra como un deber de todas las personas y de los ciudadanos, la responsabilidad de emprender acciones para la protección de los recursos naturales del país y velar por la conservación de un ambiente sano. (Art. 95, numeral 8).

En materia ambiental, la participación consiste en la incidencia de los ciudadanos en la toma de decisiones públicas que afectan al ambiente, lo cual es inherente al ejercicio del poder estatal. De esta manera, existen mecanismos que permiten integrar los conocimientos y criterios de la ciudadanía en las decisiones sobre políticas públicas determinantes de la situación del entorno (Lora, Muñoz \& Rodríguez, 2008, p. 46). De acuerdo con lo anterior, el convenio 169 de 1989 la OIT establece en sus artículos 2, 6 y 7 como punto de partida, la consulta previa como mecanismo de participación para los pueblos Indígenas y Triviales, a la hora de desarrollar planes, proyectos y actividades que los afecten de forma directa, lo anteriormente mencionado fue acogido por la Constitución de 1991 en los artículos 2, 7, 8, 40, 329 y 330.

Atendiendo a lo planteado por la Corte ( Constitucional Sentencia C-175 de 2009) la participación no se puede concebir como una intervención en la actuación administrativa que busca asegurar el derecho de defensa de quienes van a resultar afectados con la autorización de la licencia ambiental, sino que tiene una significación mayor por los altos intereses que ella busca tutelar, como son los atinentes a la definición del destino y la seguridad de la subsistencia de las referidas comunidades.
Teniendo en cuenta lo planteado por la corte constitucional en el año 1996 se ha reconocido la estrecha relación de los pueblos étnicos con la conservación, preservación y restauración del ambiente y de los recursos naturales en la medida que dichos pueblos "constituyen igualmente un recurso natural humano que se estima parte integral del ambiente, más aún cuando (...) ocupa territorios con ecosistemas de excepcionales características y valores ecológicos que deben conservarse como parte integral del patrimonio natural y cultural; además", Ramírez Moreno G. (p.59) plantea que cuando la relación hombre-selva en el departamento del Chocó, ha sido objeto de una amplia discusión por diferentes autores que han demostrado el estrecho vínculo que hay entre ellos, y lo indispensable del bosque para la población que allí subsiste, de ahí, se puede comprender la gran cantidad de permisos de aprovechamiento forestal que fueron tramitadas ante la autoridad ambiental CODECHOCÓ.

Un permiso de aprovechamiento forestal es la autorización que expide una autoridad en materia ambiental para la extracción de productos de un bosque. El Decreto 2811 de 1974, Art. 212 consagra tres tipos de permisos: único, persistente y doméstico. Para observar el enfoque que la legislación colombiana le ha dado al aprovechamiento forestal, es necesario observar el punto de partida que se estableció a partir del Decreto 2811 de 1974 (Código de los Recursos Naturales y Protección del Medio Ambiente) y el posterior desarrollo que se efectúa en esta materia en el Decreto 1791 de 1996 (Por medio de la cual se establece el régimen de aprovechamiento forestal).

Para el caso del aprovechamiento forestal único dispone: "Son aprovechamientos forestales únicos los que técnicamente se realicen en bosques localizados en suelos que deban ser destinados a usos diferentes del forestal. El permiso para aprovechamiento forestal único puede contener la obligación de dejar limpio el terreno al acabarse el aprovechamiento, 
pero no la de renovar o conservar el bosque". (Decreto 2811 de 1974, art. 218).

El Decreto 1791 de 1996, en el Art. 5 establece que los permisos de aprovechamientos forestales único son:

Los que se realizan por una sola vez, en áreas donde con base en estudios técnicos se demuestre mejor aptitud de uso del suelo diferente al forestal o cuando existan razones de utilidad pública e interés social. Los aprovechamientos forestales únicos pueden contener la obligación de dejar limpio el terreno, al término del aprovechamiento, pero no la de renovar o conservar el bosque.

Con respecto al aprovechamiento forestal, el Decreto 2811 de 1974 consagra: "son aprovechamientos forestales persistentes los que se efectúan con la obligación de conservar el rendimiento normal del bosque con técnicas silvícolas que permitan la renovación del recurso".

De igual forma, el Decreto 1791 de 1996, define en su Art. 6 lo el aprovechamiento forestal Persistente en los siguientes términos:

Los que se efectúan con criterios de sostenibilidad y con la obligación de conservar el rendimiento normal del bosque con técnicas silvícolas, que permitan su renovación. Por rendimiento normal del bosque se entiende su desarrollo o producción sostenible, de manera tal que se garantice la permanencia del bosque.

Finalmente el citado Art. 05 del Decreto 1791 de 1996, en su acápite final, se queda corto solo al manifestar que el aprovechamiento forestal doméstico, son aquellos en "Los que se efectúan exclusivamente para satisfacer necesidades vitales domésticas sin que se puedan comercializar sus productos". Sin embargo el Decreto 2811 de 1974 se hizo un poco más extenso al referirse a esta especialidad:
Son aprovechamientos forestales domésticos, los que se efectúan exclusivamente para satisfacer necesidades vitales de uso doméstico. No podrá comerciarse en ninguna forma con los productos de este aprovechamiento.

El aprovechamiento forestal doméstico deberá hacerse únicamente con permiso otorgado directamente al solicitante previa inspección, con un año de duración y con volumen máximo de veinte metros cúbicos anuales".

Herrera \& Walschburguer citado por (Giraldo, Ledezma \& Sanabria:1999,p4), plantean que el departamento del Chocó hace parte en su totalidad del importante complejo regional mundialmente conocido como "Chocó Biogeográfico", en donde se registran uno de los mayores índices de diversidad biológica del planeta también hace parte del corredor de conservación Chocó (...) reconocido como una de las más diversas biológicamente del planeta, ofreciendo un hábitat para muchas especies únicas de flora y fauna".

El Departamento de Chocó se encuentra ubicado en la esquina noroccidental de Colombia, en la región del Pacífico denominada igualmente Chocó Biogeográfico, bañado por los océanos Atlántico y Pacífico. De este modo, sus fronteras terrestres son: por el norte con la República de Panamá, el Departamento de San Andrés y Providencia; por el este con los Departamentos de Antioquia, Risaralda y Valle del Cauca, por el sur con el Departamento del Valle de Cauca. (Gobernación del Chocó, 2012).

Tiene una extensión de 46.530 Km² (11.497.813 acres), que corresponde al $4 \%$ de la extensión del país El $90 \%$ del territorio es zona especial de conservación, en la que sobresale el Tapón del Darién.

El Choco biogeográfico, más que un territorio, representa un ecosistema en donde las condiciones privilegiadas de sol, agua, luz y 
aire, elementos esenciales para la vida están presentes en forma permanente y exuberante; pero además, es una región aislada del resto de las tierras bajas de Suramérica por la cordillera de los Andes. Esta barrera natural, le genera un gran número de endemismos, en especies de: plantas, mariposas y aves, y quizás éstas últimas presentan, el mayor endemismo del mundo, aproximadamente el $25 \%$ de las especies, que allí habitan no se encuentran en ningún otro lugar del planeta. (Botero, 2010)

El aprovechamiento forestal en el Chocó, de acuerdo con el desarrollo de la investigación, en la fase de revisión de expedientes, se logró determinar que la solicitudes que se tramitan ante la entidad CODECHOCÓ, son las solicitudes de aprovechamiento forestal único y persistente, teniendo un mayor número de solicitudes, las destinadas al aprovechamiento persistente, sin embargo se debe precisar que ante la Corporación no se tramitan solicitudes de aprovechamiento forestal por ministerio de Ley debido que la Ley 70 de 1993, el principal marco normativo en tratándose de comunidades negras, en el Art. 19 consagra:

Las prácticas tradicionales que se ejerzan (...) para fines alimenticios o la utilización de recursos naturales renovables para construcción o reparación de viviendas, cercados, canoas y otros elementos domésticos para uso de los integrantes de la respectiva comunidad negra se consideran usos por ministerio de la ley y en consecuencia no requieren permiso.

Estos usos deberán ejercerse de tal manera que se garantice la persistencia de los recursos, tanto en cantidad como en calidad".

La expedición del Decreto 1791 de 1996 en el Art. 44 sigue este lineamiento cuando dispone:

Los aprovechamientos forestales que se pretendan realizar por comunidades indígenas en áreas de resguardo o reserva indígena o por las comunidades negras de que trata la Ley 70 de 1993 se regirán por las normas especiales que regulan la administración, manejo y uso de recursos naturales renovables por parte de estas comunidades (...)". Además según Hinestroza, Perea, Hinestroza y Granja (2014). Manifiestan "que en los territorios indígenas y afrocolombianos, las comunidades tienen derecho de prelación para la exploración y explotación de los recursos que se encuentren en su jurisdicción. (p. 276).

En virtud de lo anterior, el presente artículo pretende evidenciar que en el trámite de permisos de aprovechamiento forestal en el departamento del Chocó, a pesar de la existencia legal de mecanismos de participación ciudadana, el uso de mecanismos de participación no se materializa. Las comunidades sean negras o indígenas, como propietarias del territorio colectivo que tradicionalmente han ocupado de generación en generación, una vezse constituyen en territorios colectivos de comunidades negras o en resguardos indígenas, de conformidad con el artículo 8 del Decreto 1791 de 1996, establece que en los terrenos de dominio privado los permisos se otorgan a petición del propietario del predio, por lo tanto, las únicas que pueden tramitar permisos de aprovechamiento forestal en sus territorios son las comunidades negras o indígenas, lo que implica que uno de los mecanismos de participación ciudadana de tipo administrativo que frecuentemente es utilizado cuando se otorgan permisos o licencias ambientales en territorios colectivos de grupos étnicos, que más se utilice sea la consulta previa. Sin embargo, en el caso de las autorizaciones de aprovechamiento forestal en el Chocó, la mayor parte del territorio del departamento es de propiedad privada de comunidades negras e indígenas, (existen 60 consejos comunitarios y 121 cabildos indígenas ${ }^{1}$ ), por lo tanto la participación es escasa, en algunos eventos, cuando se otorgan aprovechamientos únicos para construir aeropuertos, rellenos sanitarios, etc, se puede evidenciar algunos intentos de

1 Cifra presentada por el Instituto Colombiano de Desarrollo Rural INCODER seccional Chocó mediante Oficio 20152147039 
participación, pero a través de consultas a la comunidad.

En su estructura, el presente artículo se divide en cuatro secciones: En el primer apartado se encuentra la introducción; en el segundo apartado se explica la metodología utilizada para la realización de este trabajo investigativo, el cual consistió en la recuperación bibliográfica analizando la constitución, leyes, decretos y jurisprudencia, asimismo se revisaron expedientes de permisos de aprovechamiento forestal otorgados por la autoridad ambiental del Chocó (CODECHOCÓ), durante los años de 2009 - 2013; en el tercer acápite se explica el desarrollo del proyecto, análisis de la normatividad, datos obtenidos a partir de la revisión de expedientes interpretación y discusión de la información recopilada y finalmente se presentan las conclusiones de la información recopilada.

\section{Metodología}

El desarrollo de este trabajo se realizó utilizando el método de Investigación Descriptivo propuesto por Toro y Parra (2010). A partir de la revisión de expedientes de aprovechamiento forestal otorgados por la autoridad ambiental del Chocó (CODECHOCÓ), interposición de derechos de petición, recuperación bibliográfica de fuentes formales del derecho y de doctrina, de lo anterior; se pueden determinar los requisitos para presentar la solicitud de aprovechamiento forestal, el número de autorizaciones de aprovechamiento forestal expedidas en el departamento del Chocó entre los años 2009 al 2013; identificar si en el trámite administrativo para el otorgamiento de permisos de aprovechamiento forestal que llevó a cabo la corporación CODECHOCÓ, se evidencia la participación de la ciudadanía.

\section{Desarrollo}

Desde la perspectiva internacional se ha dispuesto de una herramienta jurídica vinculante como el Convenio 169 de la Organización Internacional del Trabajo (OIT), que protege los derechos de los pueblos indígenas y tribales, en especial participación material y efectiva de estos pueblos en las decisiones que les afectan ya sea que se prevean medidas legislativas 0 administrativas, este convenio internacional fue adoptado por Colombia a través de la Ley 21 de 1991, del cual se puede resaltar en los artículos 2, 6 y 7, le impone al estado la adopción de mecanismos que permitan garantizar el derechos de los pueblos indígenas y triviales en el Estado colombiano a participar en las decisiones que los afecten, reconocidos como se expresó anteriormente, estos se encuentran en los artículos 2, 7, 8, 40, 79, 329 y 330 de la Constitución Política de Colombia.

A raíz del reconocimiento de la diversidad étnica, social y cultural en Colombia, la Carta Constitucional planteó a través del artículo transitorio 55 que en los dos (2) años siguientes a la entrada en vigencia de la constitución, se expidiera una ley que garantice los derechos de las comunidades negras asentadas en las zonas rurales ribereñas de los ríos de la Cuenca del Pacífico, la cual dio origen a la Ley 70 de 1993, en el ámbito de esta la ley es donde por primera vez se define que es una comunidad negra, en el artículo 2 numeral 5, el cual establece:

Comunidad Negra. Es el conjunto de familias de ascendencia afrocolombiana que poseen una cultura propia, comparten una historia y tienen sus propias tradiciones y costumbres dentro de la relación campo-poblado, que revelan y conservan conciencia de identidad que las distinguen de otros grupos étnicos". La expedición de esta norma es un paso importante para el reconocimiento de una nación con diversidad étnica y cultural planteado en el artículo 7 de la Constitución Política.

La Corte Constitucional a través de la sentencia T-955 de 2003, resalta lo esencial que resulta el reconocimiento de la diversidad étnica $y$ 
cultural, para la subsistencia de estos pueblos (indígenas y tribales), ya que éstos conservan y proyectan en diferentes esferas el carácter multicultural del Estado colombiano.

El Estado colombiano también ha realizado esfuerzos destinados a la garantizar uno de sus pilares fundamentales planteado en la Constitución de 1991, como es la participación ciudadana, la cual reguló a través de la Ley 134 de $1994^{2}$, esta ley estatutaria planteo la forma de participación de los ciudadanos de carácter general impersonal y abstracta, por ende, no se tuvieron en cuenta las consideraciones especiales que debía tenerse con relación a aquellos grupos de minorías étnicas, está necesidad derivó en un esfuerzo posterior el cual fue la expedición del Decreto 1320 de $\underline{1998}$, mediante el cual e reglamentó la consulta previa con las comunidades indígenas y negras para la explotación de los recursos naturales dentro de su territorio.

En ese mismo sentido la Corte Constitucional en sentencia C-030 de 2008, manifestó que en reiteradas ocasiones (Sentencias $\underline{\mathrm{C}-169 \text { de }}$ 2001, SU-383 de 2003, C-620 de 2003, T-737 de 2005, y C-208 de 2007, entre otras), esa corporación ha determinado la importancia de la consulta a estas comunidades al establecer:

De acuerdo con la jurisprudencia constitucional, en el marco del reconocimiento de la diversidad étnica y cultural como valor constitucional y fundamento de la nacionalidad colombiana (C.P. arts. 7 y 70), y en el contexto de la definición de Colombia como una república unitaria, descentralizada, con autonomía de sus entidades territoriales, democrática, participativa y pluralista (C.P. Art. 1), la Constitución Política otorga especial protección al derecho de participación de los grupos étnicos en las decisiones que los afectan.

2 Durante la ejecución del proyecto "formación en mecanismos de participación para la intervención en actuaciones administrativas en el otorgamiento de permisos ambientales 2009 - 2013" no se había expedido la ley 1757 de 2015, "Por la cual se dictan disposiciones en materia de promoción y protección del derecho a la participación democrática."
Ha destacado la Corte Constitucional que esa especial protección se traduce en el deber de adelantar procesos de consulta con las comunidades indígenas y tribales para la adopción y la ejecución de decisiones que puedan afectarles, deber que es expresión y desarrollo del artículo primero de la Constitución, que define a Colombia como una democracia participativa, del artículo $2^{\circ}$, que establece como finalidad del Estado la de facilitar la participación de todos en las decisiones que los afectan, del artículo $7^{\circ}$ Superior, que reconoce y protege la diversidad étnica y cultural, del 40-2, que garantiza el derecho de todo ciudadano a la participación democrática y del artículo 70 que considera la cultura fundamento de la nacionalidad.

En ese contexto, la Corte Constitucional ha puntualizado que, en cuanto hace a los pueblos indígenas y tribales, una de las formas de participación democrática previstas en la Carta es el derecho a la consulta, previsto de manera particular en los artículos 329 y 330 de la Constitución, que disponen la participación de las comunidades para la conformación de las entidades territoriales indígenas y para la explotación de los recursos naturales en sus territorios, y que tiene un reforzamiento en el Convenio número 169 de la OIT, aprobado por la Ley 21 de 1991, el cual está destinado a asegurar los derechos de los pueblos indígenas a su territorio y a la protección de sus valores culturales, sociales y económicos, como medio para asegurar su subsistencia como grupos humanos".

Teniendo en cuenta lo anterior, se hace necesario identificar el grado de intervención de la ciudadanía en los procesos de otorgamiento de los permisos de aprovechamiento forestal, otorgados por la autoridad ambiental CODECHOCÓ, para lo cual fue necesaria la revisión de expedientes de los permisos de aprovechamiento concedidos, para el aprovechamiento de los recursos renovables maderables, en esta fase se pudo establecer 
que si bien la normatividad colombiana hasta la fecha contempla tres tipos o clases de aprovechamiento forestal (únicos, persistentes y domésticos), cabe reiterar que ante la corporación no se encontraron evidencias de solicitudes de permisos de aprovechamiento forestal doméstico.

Los datos arrojados por la revisión de los expedientes manifiesta que de las 24 solicitudes objeto de la muestra, que fueron tramitadas ante la corporación catorce (14) corresponden a solicitudes de aprovechamiento forestal persistente y diez (10) solicitudes destinadas para la solicitud de aprovechamiento forestal único, la mayoría de las solicitudes de aprovechamiento forestal se presentaron a través de personas jurídicas (Uniones temporales, Concesionarios), los consejos comunitarios y cabildos indígenas; cabe anotar que con respecto de las solicitudes de aprovechamiento forestal persistente no se evidenció al interior de los expediente, mecanismos que hicieran inferir que hubo un consenso previo con los habitantes asentados en el territorio objeto del proyecto. (Tabla 1)

Tabla 1. Relación de los Permisos de aprovechamiento forestal Otorgados por CODECHOCÓ de 2009 -2013.

\begin{tabular}{|c|c|c|c|c|c|}
\hline Municipio & $\begin{array}{c}\text { Consejo } \\
\text { comunitario }\end{array}$ & $\begin{array}{c}\text { Resgurado } \\
\text { Indigena }\end{array}$ & $\begin{array}{c}\text { Persona } \\
\text { natural }\end{array}$ & $\begin{array}{l}\text { Persona } \\
\text { Jurídica }\end{array}$ & Total \\
\hline Bajo Baudó & & & 1 & 1 & 2 \\
\hline Carmen del Atrato & 1 & & & 1 & 2 \\
\hline Medio Atrato & 1 & & & 1 & 1 \\
\hline Tadó & & & & & 1 \\
\hline Quibdó & 2 & & 1 & & 3 \\
\hline Alto Baudó & & 1 & & & 1 \\
\hline Nuqui & 2 & & & & 2 \\
\hline Cantón de San Pablo & & & 1 & & 1 \\
\hline Rio Sucio & 4 & & 1 & & 5 \\
\hline Unguia & & & 1 & & 1 \\
\hline Novita & 1 & & & & 1 \\
\hline Unión Panamericana & 1 & & & & 1 \\
\hline Medio Baudó & & 1 & & & 1 \\
\hline Certegui & & 1 & & & 1 \\
\hline Lloró & & 1 & & & 1 \\
\hline Total & 12 & 4 & 5 & 3 & 1 \\
\hline
\end{tabular}

Al analizar la normatividad especial de las comunidades negras, Ley 70 de 1993, en artículo 24, que consagra: ..."La entidad administradora de los recursos naturales renovables reglamentará concertadamente con las comunidades negras el uso colectivo de áreas del bosque a que se refiere la presente ley, para el aprovechamiento forestal persistente", que a la par del Decreto 1745 de 1995, artículo 12 numeral 3, manifiesta que entre las funciones del representante legal del consejo comunitario está: ..."Presentar, ante la autoridad ambiental competente y ante el Ministerio de Minas y Energía, las solicitudes de aprovechamiento, exploración y explotación de recursos naturales, en beneficio de la comunidad, previa aprobación de la Junta del Consejo Comunitario; exceptuándose, los usos por ministerio de la Ley, respecto de los recursos naturales renovables". 
De lo anterior, se determina el sustento jurídico que faculta a los consejos comunitarios de presentar solicitudes de aprovechamiento forestal ante la autoridad ambiental CODECHOCÓ, y a continuación se muestra el número de consejos comunitarios existentes en el departamento del Chocó. (Tabla 2)

Tabla 2. Títulos colectivos otorgados en el departamento del Chocó.

\begin{tabular}{|c|c|c|c|}
\hline Municipio & Consejo comunitario & Resgurado Indigena & Resolución \\
\hline 1 & Alto Baudó & Bellavista Dubasa & 1219 de 01 de Junio de2000 \\
\hline 2 & Bajo Baudó & Piliza & 3367 de Diciembre de 2001 \\
\hline 3 & Bajo Baudó & Pizarro & 1122 de 16 de Mayo de 2001 \\
\hline 4 & Bajo Baudó & San Agustín de Terrón & 03369 de Diciembre 2000 \\
\hline 5 & Rio Sucio (Carmen del Darién) & Chicao & 0285 de Diciembre de 1996 \\
\hline 6 & Acandi & Rio Acandi Zona Costera Norte & 1501 de 01 Agosto de 2005 \\
\hline 7 & $\begin{array}{l}\text { Alto, Medio, Bajo Baudó, Rio } \\
\text { Quito y Cantón de San Pablo. }\end{array}$ & Rio Baudó y sus afluentes - ACABA & 1152 de 23 de Mayo de 2001 \\
\hline 8 & Carmen del Darién. & Turriquitado & 2799 de 22 de Noviembre 2000 \\
\hline 9 & $\begin{array}{c}\text { Bajo Baudó y Litoral del San } \\
\text { Juan }\end{array}$ & CONCOSTA & 1123 de 16 de Mayo de 2001 \\
\hline 10 & Bajo Baudó & Virudó & 2698 de 21 Diciembre de 2000 \\
\hline 11 & Alto Baudó & Puerto Echeverri & 1218 de 01 de Junio de 2000 \\
\hline 12 & Quibdó & Casimiro & 2429 de Diciembre de 2005 \\
\hline 13 & Acandi & Rio Acandi Seco, El Cedro y Juancho & 1499 de 01 de Agosto de 2005 \\
\hline 14 & Tadó & Mayor del Alto San Juan - ASOCASAN & 2727 de 27 de Diciembre de 2001 \\
\hline 15 & Quibdó & Guayabal & 0051 de 01 de Julio de 2003 \\
\hline 16 & Rio Sucio (Carmen del Darién) & Apartadó - Buena Vista & 0159 de 09 de Febrero de 1998 \\
\hline 17 & Rio Sucio & Cacarica & 0841 De 26 De Abril de 1999 \\
\hline 18 & Alto Baudó & San Francisco de Cugucho & 0156 de 09 de Febrero de 1998 \\
\hline 19 & Rio Sucio & Rio Salaqui & 02802 de Noviembre de 2000 \\
\hline 20 & Bajo Baudó & Cuevita & 2701 de 21 de diciembre 2001 \\
\hline 21 & Medio Baudó & Rio Pepe & 1125 de 23 de Mayo de 2000 \\
\hline 22 & Rio Sucio & La Nueva & 0289 de 13 Diciembre de 1996 \\
\hline 23 & Bajo Baudó & Pavasa & 2695 de 21 Diciembre de 2001 \\
\hline 24 & Novita & Novita & 2693 de 21 de Diciembre 2001 \\
\hline 25 & Rio Quito & San Isidro & 0157 de Febrero de 1998 \\
\hline 26 & Juradó & Juradó & 02199 de 03 Diciembre de 2002 \\
\hline 27 & Nuquí & Los Riscales & 2206 de 04 Diciembre de 2002 \\
\hline 28 & Bahía Solano & Cupica & 2700 de 21 de Diciembre 2000 \\
\hline 29 & Bajo Baudó & San Andrés de Usaragá & 3368 de 21 de Diciembre 2000 \\
\hline 30 & Certegui & Certegui & 2728 de 27 de Diciembre 2000 \\
\hline 31 & Acandi & Rio Tolo Zona Costera Sur & 1502 de 01 Agosto de 2005 \\
\hline 32 & Rio Sucio & Dos Bocas & 028813 de Diciembre de 1996 \\
\hline 33 & Carmen del Darién & La Grande & 2806 de 22de Noviembre 2000 \\
\hline 34 & Rio Sucio & La Madre & 0286 de Diciembre de 1996 \\
\hline 35 & Istmina y Medio San Juan & Istmina y Medio San Juan & 1176 de Julio de 2002 \\
\hline
\end{tabular}




\begin{tabular}{|c|c|c|c|}
\hline Municipio & Consejo comunitario & Resgurado Indigena & Resolución \\
\hline 36 & Cantón de San Pablo & ACISANPI & 2694 de Diciembre de 2001 \\
\hline 37 & Condoto & Condoto & 1177 de 16 de Julio de 2002 \\
\hline 38 & Rio Sucio & Clavellino & 0290 de 13 de Diciembre 1996 \\
\hline 39 & Rio Quito & Villa Conto & 0160 de 09 de Febrero de 1998 \\
\hline 40 & $\begin{array}{l}\text { Quibdó, Atrato, Rio Quito, Medio } \\
\text { Atrato, Bojayá, Vigía del Fuerte, } \\
\text { Murindo y Urrao de Antioquia. }\end{array}$ & Asociación Integral del Atrato - ACIA. & 4566 de 29 de Diciembre de 1997 \\
\hline 41 & Rio Sucio & Pedeguita y Mancilla & 2804 de 22 de Noviembre 2000 \\
\hline 42 & Bajo Baudó & Villa María de Purricha & 1129 de 23 de Mayo de 2000 \\
\hline 43 & Bajo Baudó & Siviru & 1144 de 14 de Mayo de 2007 \\
\hline 44 & Carmen del Darién & Rio Jiguamiando & 2801 de Noviembre de 2000 \\
\hline 45 & Carmen del Darién & Rio Curvaradó & 2809 de 22 de Noviembre 2000 \\
\hline 46 & Rio Sucio & Truando Medio & 3366 de 21 de Diciembre 2000 \\
\hline 47 & Carmen del Darién & Montaño & 2807 de 22 de Noviembre 2000 \\
\hline 48 & Rio Sucio & Bocas De Taparal & 2803 de Noviembre de 2000 \\
\hline 49 & Carmen del Darién & Rio Domingodó & 2803 de 22 Noviembre 2000 \\
\hline 50 & Rio Sucio & La Larga Tumaradó & 2805 de Noviembre de 2000 \\
\hline 51 & Carmen del Darién & $\begin{array}{c}\text { Vigía de Curvaradó y Santa Rosa de } \\
\text { Limón. }\end{array}$ & 2808 de Noviembre de 2000 \\
\hline 52 & Unguia & Bajo Atrato & 0048 de 21 de Julio de 2003 \\
\hline 53 & Rio Quito & Paimadó & 2726 de 27 de Diciembre 2001 \\
\hline 54 & $\begin{array}{l}\text { Litoral del San Juan, Istmina y } \\
\text { Novita. }\end{array}$ & ACADESAN & 2702 de 21 de Diciembre 2001 \\
\hline 55 & Bahía Solano y Jurado. & Los Delfines & 002200 de Diciembre de 2002 \\
\hline 56 & Unión Panamericana & COCOMAUPA & 2723 de 27 de Diciembre 2001 \\
\hline 57 & Atrato, Lloro, Bagadó, Certegui. & COCOMAPOCA & 02425 de 19 Septiembre 2011 \\
\hline 58 & Rio Sucio & Rio Quiparadó & 2798 de Noviembre de 2000 \\
\hline 59 & Lloro & Consejo Comunitario Integ. De Lloro & 2919 de 21 Diciembre de 2012. \\
\hline 60 & Atrato & Consejo comunitario de la Molana & 00631 de Marzo de 2015 \\
\hline
\end{tabular}

Con la expedición de la carta política de 1991, se tomaron medidas concernientes a la reivindicación de los derechos de las minorías étnicas, el Estado colombiano también ha legislado para proteger los derechos de las comunidades indígenas, con respecto a las normas especiales para los resguardos indígenas, la Corte Constitucional a través de la sentencia C-139 de 1996, manifiesta que:

El Constituyente de 1991 con el fin de proteger la integridad territorial y cultural de los pueblos indígenas estableció la propiedad colectiva de los resguardos y de las tierras comunales de las etnias asignándoles, entre otros, el carácter de inajenables, de manera que no pueden ser objeto de venta o transacción alguna por parte de ninguno de los miembros que conforman la comunidad indígena. Quiso así el Constituyente defender las tierras de los pueblos indígenas como colectividad sujeta a tratamiento especial.

La Corte Constitucional en la sentencia T-380 de 1993 establece: 
Laautonomía de las autoridadesindígenas en el manejo de sus propios asuntos, en especial respecto del aprovechamiento de los recursos naturales, debe ser ejercida con plena responsabilidad. En favor de la comunidad indígena siempre podrá aducirse la doctrina ultra vires frente a actuaciones de sus autoridades que hayan dispuesto ilegal o arbitrariamente de las riquezas naturales comprendidas en su territorio, y a las cuales por lo tanto se las debe despojar de todo poder vinculante. (Tabla 3. Ver anexo)

Se hace necesario la observancia de la normatividad colombiana en materia forestal a través de la Ley 93 de 1931, por la cual se fomenta la explotación de los recursos forestales, El Decreto 2278 de 1953 que dicta medidas sobre cuestiones forestales, Ley 2 de 1959 , sobre economía forestal de la Nación y recursos renovables, Decreto 2811 de 1974 por el cual se dicta el Código Nacional de Recursos Naturales y de Protección al Medio Ambiente, Decreto 877 de 1976 por el cual se señalan prioridades referentes a los diversos usos del recurso forestal, a su aprovechamiento y al otorgamiento de permisos y concesiones y se dictan otras disposiciones, Decreto 622 de 1977 que reglamenta parcialmente el Capítulo V, Título II, Parte XIII, Libro II del DecretoLey 2811 de 1974 sobre "Sistema de Parques Nacionales".

Al igual que los Decreto 1741 de 1978 que reglamenta parcialmente la Ley 23 de 1973, el Decreto 2811 de 1974 y los Decretos 2349 de 1971 y 133 de 1976, en lo relacionado con la creación de un Área de Manejo Especia, Ley 37 de 1989, por la cual se dan las bases para estructurar el Plan Nacional de Desarrollo forestal, Ley 99 de 1993, por la cual se crea el Ministerio del Medio Ambiente, se reordena el Sector Público encargado de la gestión y conservación del medio ambiente y los recursos naturales renovables, se organiza el Sistema Nacional Ambiental, SINA, y se dictan otras disposiciones.
Y finalmente la Ley 139 de 1994 que crea el certificado de incentivo forestal y se dictan otras disposiciones, Decreto 1824 de 1994 por el cual se reglamenta parcialmente la (Ley 139 de 1994), Decreto 2915 de 1994 por el cual se organiza la Unidad Administrativa Especial del Sistema de Parques Nacionales Naturales, se asignan funciones y se dictan otras disposiciones. Decreto 1791 de 1996, mencionado al principio, por medio del cual se establece el régimen de aprovechamiento forestal, Decreto 900 de 1997 que reglamenta el certificado de incentivo forestal, Decreto 1498 de 2008 que reglamenta el parágrafo $3^{\circ}$ del artículo $5^{\circ}$ de la Ley 99 de 1993 y el artículo 2 de la Ley 139 de 1994.

Una vez hecha la recuperación bibliográfica en materia de aprovechamiento forestal en Colombia, se procedió a verificar en la fase de revisión de expedientes si se cumplen los requisitos legales al momento de realizar los trámites aprovechamiento forestal único y aprovechamiento persistente, en estos expedientes se evidenció que en los trámites para el otorgamiento de autorizaciones de aprovechamiento forestal único, todas presentaron la figura de la consulta previa con las comunidades arraigadas en el territorio donde se va a ejecutar la actividad, además de los requisitos establecidos por el artículo 13 del Decreto 1791 de 1996, que en materia de solicitud de permisos de aprovechamiento forestal único establece que para tramitar aprovechamiento forestal único de bosques naturales ubicados en terrenos de dominio público se requiere, por lo menos, que el interesado presente ante la Corporación en cuya jurisdicción se encuentre al área objeto de aprovechamiento:

a) Solicitud formal.

b) Estudio técnico que demuestre una mejor aptitud de uso del suelo diferente al forestal.

c) Plan de aprovechamiento forestal, influyendo la destinación de los productos forestales y las medidas de compensación. 
Sin embargo con respecto el aprovechamiento forestal persistente, en la revisión de expedientes no se encontró evidencia de la intervención ciudadana en el trámite de los mismos, solo se encuentra los requisitos planteados por el Decreto 1791 de 1996 en el Art. 6, el cual establece:

Para adelantar aprovechamientos forestales persistentes de bosques naturales ubicados en terrenos de dominio público se requiere, por lo menos, que la zona se encuentre dentro del área forestal productora o protectora-productora alinderada por la Corporación respectiva y que los interesados presenten, por lo menos: a) Solicitud formal. b) Acreditar capacidad para garantizar el manejo silvicultura, la investigación y la eficiencia en el aprovechamiento y en la transformación. c) Plan de manejo forestal.

Sin embargo para el objeto de esta investigación sería importante que la ciudadanía en general (no sólo las comunidades negras) pudiera intervenir en el trámite de otorgamiento de estos permisos, la Corte Constitucional por medio de la sentencia C-150 de 2015, manifiesta que: "la Constitución fija como una forma de participación el ejercicio de las acciones administrativas o judiciales requeridas para el control de las actividades a cargo del Estado o para la efectividad de los derechos colectivos". En concordancia en materia de participación la Ley 99 de 1993. Art. 69 establece:

Cualquier persona natural o jurídica o privada, sin necesidad de demostrar interés jurídico alguno, podrá intervenir en las actuaciones administrativas iniciadas para la expedición, modificación o cancelación de permisos o licencias de actividades que afecten o puedan afectar el medio ambiente o para la imposición o revocación de sanciones por el incumplimiento de las normas y regulaciones ambientales.

Es de anotar que después de un análisis del Decreto 1791 del 1996, no dispone al interior de su articulado, mecanismos que permitan que la comunidad pueda participar, y/o emitir un concepto durante el proceso de otorgamiento del permiso de aprovechamiento forestal, partiendo de la premisa que son ellos los directos afectados con dicha autorización.

En el proceso de solicitud de la autorización de aprovechamiento forestal persistente se puede inferir que los titulares de aquellos permisos de aprovechamiento forestal es la misma comunidad que a través de los consejos comunitarios y/o cabildos indígenas quienes solicitan los permisos de aprovechamiento forestal ante la respectiva autoridad ambiental, y que estas formas de organización facultadas por la ley realizan los trámites para la obtención de los permisos, y de esta manera aprovechar las diferentes especies madereras de la región; pero no hay intervención de otros que teniendo en cuenta que todos tenemos derecho a gozar de un ambiente sano, consideren de interés conocer sobre los permisos y recursos naturales que están siendo aprovechados por las comunidades. La participación no es sólo para los grupos étnicos, es para toda la comunidad, para todos los ciudadanos.

Asimismo, se observó que, es necesario fortalecer las actividades de intervención de la Procuraduría Delegada para asuntos Ambientales y Agrarios del Chocó dentro del proceso otorgamiento de permisos de aprovechamiento forestal ya que en los expedientes se encontró que solo 1 de las 24 solicitudes revisadas, evidenciaba acción de esta entidad ante la autoridad ambiental CODECHOCÓ, el cual consistió en un recurso de reposición en contra del acto administrativo que otorgaba un permiso de aprovechamiento forestal porque consideraba que no se había realizado la consulta previa y que solo hubo una comunicación a la comunidad sobre las nociones generales del proyecto. 


\section{Conclusiones}

El Estado colombiano se ha preocupado en gran medida por la protección del medio ambiente, esto se puede evidenciar por la cantidad de artículos de la Constitución Política que se refieren en su protección y al extenso cuerpo normativo que lo complementan, entre ellos leyes, decretos y jurisprudencia, a la par de la preocupación del Estado por el medio ambiente, se encuentra el reconocimiento de la diversidad étnica y cultural (Art. 7), de los pueblos conocidos como "minorías étnicas" que han habitado ancestralmente los territorio colombiano para quienes se han expedido normas que buscan la reivindicación de sus derechos a la propiedad ya la autodeterminación de sus pueblos en las decisiones que puedan afectarlos (Ley 21 de 1991, Ley 70 de 1993, Decreto 1745 de 1995, Decreto 2164 de 1995 y Decreto 1320 de 1998).

Gracias a la diversidad étnica, ambiental y cultural que se encuentra en departamento del Chocó y a la estrecha relación de estos con el medio ambiente, se hace necesario que los ciudadanos o comunidades conozcan la existencia de los mecanismo de participación ciudadana que permitan propender por la reducción, preservación y protección del medio ambiente.

La participación es importante en los proceso de otorgamiento de permisos de aprovechamiento forestal, como se manifiesta en materia de participación ciudadana que ha tenido Colombia a través del desarrollo jurisprudencial que ha realizado la Corte Constitucional, como establece en sentencia C-891 de 2002, en la cual manifiesta que para garantizar la efectividad del derecho a participar en las decisiones que afectan o puedan llegar a afectar los legítimos intereses de un grupo determinado, es responsabilidad de las entidades estatales suministrar a las personas oportunamente toda la información que no goce de reserva constitucional o legal; y que además esta información debe ser completa, consistente, coherente, verificable, comparable, contextualizada, diáfana y siempre oportuna y que igualmente deben asumir la promoción, creación y fomento de las condiciones idóneas a la discusión pública de los temas pertinentes; proponiendo una participación ciudadana donde la discusión que se presente sea constructiva basada en el respeto de los criterios expuestos por los interlocutores institucionales y privados.

Dada la importancia en que reviste el ejercicio de este derecho fundamental para la comunidad, porque le permite conocer y participar de forma activa en dicho proceso, teniendo en cuenta que serán ellos los directos afectados o beneficiarios en la realización de proyectos, obras o actividades que se desarrollen en sus comunidades y de esta forma como ese establece en la sentencia U-039 de 1997 la participación estaría reducida solamente a una intervención en actuaciones administrativas que buscan asegurar el derecho de defensa de quienes van a resultar afectados con la autorización del aprovechamiento forestal emitido por la autoridad ambiental, sino que sino que además trasciende por el alcance de los intereses que busca tutelar, que en últimas son los pertinentes al futuro y subsistencia de las comunidades asentadas en el lugar donde se ejecute el proyecto.

Según los datos obtenidos en los expedientes revisados, no se logró observar en las solicitudes de aprovechamiento forestal persistente que indique que la comunidad asentada en los territorios donde se pretende realizar aprovechamiento forestal se les comunique, publique o haya una concertación de la realización de la actividad de una forma anticipada, por consiguiente no existe intervención de la comunidad en el trámite de otorgamiento de permisos de aprovechamiento forestal, que solicitan los consejos comunitarios y/o cabildos indígenas ante la Corporación CODECHOCÓ, pero por el contrario, en su 
totalidad las solicitudes de aprovechamiento forestal único presentan la consulta previa como mecanismo de participación ciudadana.

\section{Referencias}

Amaya Navas, O. D. (2010). La Constitución Ecológica de Colombia. Segunda Edición, Bogotá: Universidad Externado de Colombia.

Botero Chica, C. (2010). El Chocó Biogeográfico, un tesoro de la naturaleza. Recuperado de: [link].

El derecho de prelación de las comunidades negras en la explotación minera en el municipio de Condoto - Chocó".

El derecho de las Comunidades Afrocolombianas a la Consulta Previa, Libre e Informada" Recuperado de: [link]

Giraldo Álvarez, L. A., Guido Ledezma. M. A. \& Sanabria Botero. J. R. (2012). El transporte: la locomotora del desarrollo del pacífico norte colombiano. Primera Edición. Quibdó. Universidad Tecnológica del Chocó "Diego Luis Córdoba"

Gobernación Del Choco "Bases Del Plan De Desarrollo 2012- 2015”. Recuperado de: [link].

Hinestroza Cuesta, L., Hinestroza Ramírez, W. E., Perea Zapata, M., Granja Murillo , M. L. (2014). El derecho de prelación de las comunidades negras en la explotación minera en el municipio de Condoto-Chocó" Recuperado de: https://aprendeenlinea.udea.edu.co/revistas/index.php/red/ article/download/21665/17854

Jimeno Santoyo, M., Sotomayor, M. L., Valderrama, L. M. (1995). "Chocó: diversidad cultural y medio ambiente". Recuperado de: [link].
Lora, A. M., Muñoz, L. M., Rodríguez, G. A. (2008). Manual de Acceso a la Información y a la Participación Ambiental en Colombia. Primera edición. Bogotá: Universidad del Rosario

Toro, I. D., Parra, R. D., (2010). Fundamentos epistemológicos de la investigación y la metodología de la investigación cualitativa/cuantitativa. Primera Edición. Medellín: Fondo Editorial-Universidad EAFIT.

\section{Leyes}

República de Colombia. (1890). Ley 89 de 1890. "Por medio de la cual se determina la manera cómo deben ser gobernados los salvajes que vayan reduciéndose a la vida civilizada". Versión digital disponible en: [link]. (Enero de 2015).

República de Colombia. (1991). Asamblea Nacional Constituyente. Constitución Política de 1991. Bogotá, Colombia. Versión digital disponible en: [link]. (Enero de 2015)

República de Colombia. (1991). Ley 21 de 1991. "Por medio de la cual se aprueba el Convenio número 169 sobre pueblos indígenas y tribales en países independientes, adoptado por la 76a. reunión de la Conferencia General de la O.I.T”. Versión digital disponible en: [link]. (Marzo de 2015)

República de Colombia. (1931). Ley 93 de 1931. "Por medio de la cual se fomenta la explotación de productos forestales". Colombia. Versión digital disponible en: http://documentacion.ideam.gov. co/openbiblio/bvirtual/005574/3/LY009331.RTF

República de Colombia. (1959). Ley $2^{\mathrm{a}}$ de 1959, Por medio de la cual se dictan nomas sobre economía forestal de la Nación y conservación de recursos naturales renovables. Colombia. Versión digital disponible en: [link]. (Marzo de 2015) 
República de Colombia. (1989). Ley 37 de 1989. Por medio de la cual se dan las bases para estructurar el Plan Nacional de Desarrollo Forestal y se crea el Servicio Forestal. Colombia. Versión digital disponible en: [link]. (Abril de 2015)

República de Colombia. (1993). Ley 70 de 1993. Por medio de la cual se desarrolla el artículo transitorio 55 de la Constitución Política. Colombia. Versión digital disponible en: [link]. (Abril de 2015)

República de Colombia. (1993). Ley 99 de 1993. "Por medio de la cual se crea el Ministerio del Medio Ambiente, se reordena el Sector Público encargado de la gestión y conservación del medio ambiente y los recursos naturales renovables, se organiza el Sistema Nacional Ambiental, SINA, y se dictan otras disposiciones. Colombia. Versión digital disponible en: [link]. (Mayo de 2015)

República de Colombia. (1994). Ley 139 de 1994. Por la cual se crea el Certificado de Incentivo Forestal y se dictan otras disposiciones. Colombia. Versión digital disponible en: [link]. (Abril de 2015)

República de Colombia. (1994). Ley 134 de 1994, Por medio de la cual se dictan normas sobre mecanismos de participación ciudadana.

\section{Decretos}

República de Colombia. (1953). Decreto 2278 de 1953, "Por el cual se dictan medidas sobre cuestiones forestales". Colombia. Recuperado de: [link]

República de Colombia. (1974). Decreto 2811 de 1974, "Por el cual se dicta el Código Nacional de Recursos Naturales Renovables y de Protección al Medio Ambiente". Colombia. Recuperado de: [link]
República de Colombia. (1976). Decreto 877 de 1976, "Por el cual se señalan prioridades referentes a los diversos usos del recurso forestal, a su aprovechamiento y al otorgamiento de permisos y concesiones y se dictan otras disposiciones". Colombia. Recuperado de: [link]

República de Colombia. (1977). Decreto 622 de 1977, "Por el cual se reglamentan parcialmente el capítulo V, título II, parte XIII, libro II del Decreto- Ley número 2811 de 1974 sobre «sistema de parques nacionales»; la Ley 23 de 1973 y la Ley 2a de 1959”. Colombia. Recuperado de: [link]

República de Colombia. (1978). Decreto 1741 de 1978, "Por el cual se reglamentan parcialmente la Ley 23 de 1973, el Decreto Ley 2811 de 1974 y los Decretos 2349 de 1971 y 133 de 1976, en lo relacionado con la creación de un Área de Manejo Especial". Colombia. Recuperado de: [link]

República de Colombia. (1993). Decreto 1088 de 1993, "Por el cual se regula la creación de las asociaciones de Cabildos y/o Autoridades Tradicionales Indígenas". Colombia. Recuperado de: [link]

República de Colombia. (1994). Decreto 1824 de 1994, "Por el cual se reglamenta parcialmente la Ley 139 de 1994". Colombia. asignan funciones y se dictan otras disposiciones". Colombia. Recuperado de: [link]

República de Colombia. (1994). Decreto 2915 de 1994, "Por el cual se organiza la Unidad Administrativa Especial del Sistema de Parques Nacionales Naturales, se asignan funciones y se dictan otras disposiciones". Colombia. Recuperado de: [link]

República de Colombia. (1995). Decreto 1745 de 1995. "Por el cual se reglamenta el Capítulo III de la Ley 70 de 1993, se adopta el proce- 
dimiento para el reconocimiento del derecho a la propiedad colectiva de las "Tierras de las Comunidades Negras" y se dictan otras disposiciones". Colombia. Recuperado de: [link]

República de Colombia. (1996). Decreto 1396de 1996, "Por medio del cual se crea la Comisión de Derechos Humanos de los Pueblos Indígenas y se crea el programa especial de atención a los Pueblos Indígenas". Colombia. Recuperado de: [link]

República de Colombia. (1996). Decreto 1791 de 1996, "Por medio del cual se establece el régimen de aprovechamiento forestal". Colombia. Recuperado de: [link]

República de Colombia (1997). Decreto 900 de 1997, "Por el cual se reglamenta el Certificado de Incentivo Forestal para Conservación". Colombia. Recuperado de: [link]

República de Colombia (1998). Decreto 1320 de 1998, "Por la cual se reglamenta la consulta previa con las comunidades indígenas y negras para la explotación de los recursos naturales dentro de su territorio". Colombia. Recuperado de: [link]

República de Colombia. (2002). Decreto 1745 de 2002, "Por el cual se reglamenta parcialmente la Ley 715 de 2001 ”. Colombia. Recuperado de: [link]

República de Colombia (2008). Decreto 1498 de 2008, "Por el cual se reglamenta el parágrafo $3^{\circ}$ del artículo $5^{\circ}$ de la Ley 99 de 1993 y el artículo $2^{\circ}$ de la Ley 139 de 1994". Colombia. Recuperado de: [link]

\section{Jurisprudencia}

República de Colombia. Corte Constitucional. (1993). Sentencia T-380 de 1993. República de Colombia. Magistrado ponente: Dr. Eduardo Cifuentes Muñoz Recuperado de: [link]
República de Colombia. Corte Constitucional. (1996). Sentencia C-139 de 1996. República de Colombia. Magistrado Ponente: Dr. Carlos Gaviria Díaz. Recuperado de: [link]

República de Colombia. Corte Constitucional. (1996). Sentencia T-349 de 1996. República de Colombia. Magistrado Ponente: Dr. Carlos Gaviria Díaz. Recuperado de: [link]

República de Colombia. Corte Constitucional. (1997). Sentencia SU-039 de 1997. República de Colombia. Magistrado ponente: Dr. Antonio Barrera Carbonell Recuperado de: [link]

República de Colombia. Corte Constitucional. (1997). Sentencia T-523 de 1997. República de Colombia. Magistrado Ponente: Dr. Carlos Gaviria Díaz. Recuperado de: [link]

República de Colombia. Corte Constitucional. (2001). Sentencia C-169 de 2001. República de Colombia. Magistrado Ponente: Dr. Carlos Gaviria Díaz. Recuperado de: [link]

República de Colombia. Corte Constitucional. (2002). Sentencia C-891 del 2002. República de Colombia Magistrado Ponente: Dr. Jaime Araujo Rentería. Recuperado de: [link]

República de Colombia. Corte Constitucional. (2003). Sentencia T-955 de 2003. República de Colombia. Magistrado Ponente: Dr. Álvaro Tafur Galvis Recuperado de: [link]

República de Colombia. Corte Constitucional. (2003). Sentencia SU-383 de 2003. República de Colombia. Magistrado Ponente: Dr. Álvaro Tafur Galvis. Recuperado de: [link]

República de Colombia. Corte Constitucional. (2003). Sentencia C-620 de 2003. República de Colombia. Magistrado Ponente: Dr. Marco Gerardo Monroy Cabra. Recuperado de: [link] 
República de Colombia. Corte Constitucional. (2005). Sentencia T-737 de 2005. República de Colombia. Magistrado Ponente: Dr. Álvaro Tafur Galvis. Recuperado de: [link] "

República de Colombia. Corte Constitucional. (2007). Sentencia C-208 de 2007. República de Colombia. Magistrado Ponente: Dr. Rodrigo Escobar Gil. Recuperado de: [link]

República de Colombia. Corte Constitucional. (2008). Sentencia C-030 de 2008. República de Colombia. Magistrado Ponente: Dr. Rodrigo Escobar Gil. Recuperado de: [link]

República de Colombia. Corte Constitucional. (2008). Sentencia C-461 de 2008. República de Colombia. Magistrado Ponente: Dr. Manuel José Cepeda Espinosa. Recuperado de: [link]
República de Colombia. Corte Constitucional. (2009). Sentencia C-175 de 2009. República de Colombia. Magistrado Ponente: Dr. Luis Ernesto Vargas Silva. Recuperado de: [link]

República de Colombia. Corte Constitucional. (2009). Sentencia T 769 de 2009. República de Colombia. Magistrado Ponente: Dr. Nilson Pinilla Pinilla. Recuperado de: [link]

República de Colombia. Corte Constitucional. (2015). Sentencia C-150 de 2015. República de Colombia. Magistrado Ponente: Mauricio González Cuervo. Recuperado de: [link] 


\section{Anexo Tabla 3}

Tabla 3. Relación de Resguardos Indígenas constituidos en el departamento del Chocó.

\begin{tabular}{|c|c|c|c|}
\hline Municipio & Municipio & Nombre del Resgurado & Resolución de constitución \\
\hline 1 & Medio Baudó & Puerto Libre del Río Pepe & $N^{\circ} 050$ de $10 / 07 / 1989$ \\
\hline 2 & Bajo Baudó (Medio Baudó) & Trapiche del Río Pepe & $N^{\circ} 051$ de 10/07/1989 \\
\hline 3 & Bajo Baudó (Medio Baudó) & Quebrada Querá & $N^{\circ} 016$ de $23 / 06 / 1992$ \\
\hline 4 & Medio Baudó & Chigorodo Memba & $\mathrm{N}^{\circ} 015$ de $10 / 04 / 2003$ \\
\hline 5 & Bojaya & Pachicora, chicué y punto Alegre & $\mathrm{N}^{\circ} 040$ de $30 / 11 / 1998$ \\
\hline 6 & Nuquí & Río Nuqui & $\mathrm{N}^{\circ} 013$ de $21 / 04 / 1982$ \\
\hline 7 & Bahía Solano & Rios Valle, Posamansa y Boroboro & Nº30 de 08/05/1984 \\
\hline 8 & Carmen del Darien & Urada Jiguamiandó - Guayabal & $\mathrm{N}^{\circ} 007$ de $22 / 07 / 2003$ \\
\hline 9 & Quibdó & Caimanero de Jampapa & $N^{\circ} 027$ de $03 / 04 / 1987$ \\
\hline 10 & Quibdó & Río Ichó Quebrada Baratudo & $N^{\circ} 021$ de $24 / 05 / 1989$ \\
\hline 11 & Quibdó & El Veintiuno & $N^{\circ} 003$ de $25 / 01 / 1994$ \\
\hline 12 & Quibdó & Guarando, Carrizal & $N^{\circ} 035$ de $31 / 05 / 1999$ \\
\hline 13 & Bajo Baudó & La Jagua,Guachacal Pitalito & $N^{\circ} 058$ de 29/09/1992 \\
\hline 14 & Bajo Baudó, Litoral San Juan & Rio Orupa & $N^{\circ} 021$ de $18 / 03 / 1987$ \\
\hline 15 & Bajo Baudó & Bajo Grande & $N^{\circ} 012$ de 28/06/2001 \\
\hline 16 & Quibdó (Medio Atrato) & Paina & $N^{\circ} 054$ de 23/12/1998 \\
\hline 17 & Bajo Baudó & Ordó, sivirú, Agua clara & $N^{\circ} 057$ de 29/09/1992 \\
\hline 18 & Bajo Baudó & Do Imama, Tumá y Bella Luz & $N^{\circ} 014$ de $10 / 04 / 2003$ \\
\hline 19 & Juradó & Santa Marta Rio Curiche & $N^{\circ} 020$ de $18 / 03 / 1987$ \\
\hline 20 & Bojaya & Rios Tungina y Apartadó & $N^{\circ} 074$ de $19 / 11 / 1990$ \\
\hline 21 & Bojaya & Rio Bojaya & $N^{\circ} 023 \mathrm{de} 03 / 12 / 1981$ \\
\hline 22 & Rio Sucio Carmen del Darien & Jagual Rio Chintado & $N^{\circ} 136$ de $03 / 12 / 1980$ \\
\hline 23 & Acandi & Chidima Tolo & $N^{\circ} 005$ de $20 / 02 / 2001$ \\
\hline 24 & Litoral del san Juan -B/ventura & Burujó Unión San Bernardo & $N^{\circ} 012$ de 03/05/1983 \\
\hline 25 & Carmen del Atrato & El Doce Quebrada Borbollón & $N^{\circ} 016$ de $21 / 04 / 1982$ \\
\hline 26 & Nuqui y alto Baudo & Alto Baudó Jurubira - Chori & $\mathrm{N}^{\circ} 015 \mathrm{de} 21 / 04 / 1982$ \\
\hline 27 & Bojaya & Buchadó - Amparrado & $N^{\circ} 001$ de $15 / 01 / 1980$ \\
\hline 28 & Carmen del Atrato & Abejero & $N^{\circ} 079$ de 09/12/1999 \\
\hline 29 & Unguia & Rio Cuti & $N^{\circ} 103$ de $27 / 07 / 1982$ \\
\hline 30 & $\begin{array}{l}\text { Medio San Juan y Litoral del } \\
\text { San Juan }\end{array}$ & Puadó, Mataré y la Lerma & $N^{\circ} 039$ de $03 / 07 / 1986$ \\
\hline 31 & Bajo Baudó & Rio Purricha & $N^{\circ} 026$ de13/07/1992 \\
\hline 32 & Bajo Baudó & Pavasa- Jella & $N^{\circ} 077$ de $14 / 04 / 1993$ \\
\hline 33 & Certegui & Pared-Parecito & $N^{\circ} 018$ de $10 / 04 / 1993$ \\
\hline 34 & Carmen del Atrato & Rio la Playa & $N^{\circ} 072$ de $14 / 04 / 1993$ \\
\hline 35 & Quibdó (Medio Atrato) & Beté-Auro Beté-Auro del Buey & $N^{\circ} 016$ de 03/05/1983 \\
\hline 36 & Rio Sucio Carmen del Darien & Mamey de Dipurdú & $N^{\circ} 020$ de $10 / 04 / 2003$ \\
\hline 37 & Rio Sucio & Salaquí-Pavarandó & $N^{\circ} 015$ de $03 / 05 / 1983$ \\
\hline
\end{tabular}




\begin{tabular}{|c|c|c|c|}
\hline Municipio & Municipio & Nombre del Resgurado & Resolución de constitución \\
\hline 38 & Tadó & Mondo-Mondocito & $N^{\circ} 072$ de $29 / 08 / 1988$ \\
\hline 39 & Litoral del San Juan & Rio Taparal & $N^{\circ} 070$ de $06 / 11 / 1985$ \\
\hline 40 & Litoral del San Juan -B/ventura & Papayo & $N^{\circ} 102$ de $15 / 12 / 1981$ \\
\hline 41 & Lloró & Río Munbú & $N^{\circ} 063$ de $21 / 09 / 1983$ \\
\hline 42 & Lloró & Wanchirado & $\mathrm{N}^{\circ} 071$ de $12 / 12 / 1992$ \\
\hline 43 & Lloró & Gegora-Quipara-Tiravenado y Jiguado & $\mathrm{N}^{\circ} 011$ de 28/06/2001 \\
\hline 44 & Lloró & Tokolloro & $N^{\circ} 009$ de $20 / 02 / 2001$ \\
\hline 45 & Lloró & Hurtado-tagavera & $N^{\circ} 072$ de $12 / 12 / 1992$ \\
\hline 46 & Lloró & Lanas & $N^{\circ} 003$ de 25/01/1984 \\
\hline 47 & Bojaya & Opogodó- Doguado & $N^{\circ} 064$ de $01 / 10 / 1986$ \\
\hline 48 & Bojaya & Alto Rio Bojaya & $N^{\circ} 048$ de $10 / 07 / 1986$ \\
\hline 49 & Bojaya & Alto Rio Cuia & $N^{\circ} 049$ de 01/10/1986 \\
\hline 50 & Bagadó & Tahami del Andagueda & $N^{\circ} 185$ de $13 / 11 / 1979$ \\
\hline 51 & Juradó & Guayabal de Partadó & $N^{\circ} 054$ de $24 / 07 / 1987$ \\
\hline 52 & Litoral del San Juan -B/ventura & Cabeceras- Puerto Pizario & $\mathrm{N}^{\circ} 013$ de $03 / 05 / 1983$ \\
\hline 53 & Bajo Baudó (Medio Baudó) & Torreidó Chimani & $N^{\circ} 062$ de $21 / 09 / 1983$ \\
\hline 54 & Lloró & Guadualito & $N^{\circ} 017$ de $10 / 12 / 2002$ \\
\hline 55 & Quibdó (Medio Atrato) & Alto Rio Buey & $N^{\circ} 023$ de 10/04/1986 \\
\hline 56 & Bajo Baudó & Santa Rosa de ljua & $N^{\circ} 056$ de $26 / 09 / 1992$ \\
\hline 57 & Bajo Baudó & El Piñal & $\mathrm{N}^{\circ} 006$ de $20 / 02 / 2001$ \\
\hline 58 & Bajo Baudó (Medio Baudó) & $\begin{array}{l}\text { Santa Cecilia de la Quebrada oro } \\
\text { Chocó }\end{array}$ & $\mathrm{N}^{\circ} 049$ de $10 / 07 / 1989$ \\
\hline 59 & Bajo Baudó & Bellavista Unión Pitalito & $N^{\circ} 040$ de $15 / 08 / 1984$ \\
\hline 60 & Litoral del San Juan -B/ventura & Nuevo Pitalito & $N^{\circ} 055$ de 29/09/1992 \\
\hline 61 & Quibdó (Medio Atrato) & Rio Bebarama & $N^{\circ} 066$ de 06/08/1988 \\
\hline 62 & Alto Baudó & Agua Clara Bella Luz & $N^{\circ} 023$ de $10 / 04 / 1989$ \\
\hline 63 & Urrao Antioquia & Andabú & $\mathrm{N}^{\circ} 027$ de $31 / 05 / 1999$ \\
\hline 64 & Rio Sucio (Carmen del Darien) & Rio Domingodó & $\mathrm{N}^{\circ} 035$ de $21 / 07 / 1983$ \\
\hline 65 & Litoral del San Juan & Tiosidilio & $N^{\circ} 011$ de $03 / 05 / 1983$ \\
\hline 66 & Litoral del San Juan & Togoroma & $\mathrm{N}^{\circ} 107$ de $15 / 12 / 1981$ \\
\hline 67 & Litoral del San Juan & Buena Vista & $N^{\circ} 054$ de 29/09/1992 \\
\hline 68 & Litoral del San Juan & Rio Pichimá & $N^{\circ} 071$ de 06/09/1985 \\
\hline 69 & Litoral del San Juan -B/ventura & Chachajo & $N^{\circ} 0103$ de 15/12/1981 \\
\hline 70 & Litoral del San Juan & Docordó-Balsalito & $N^{\circ} 0106$ de $15 / 12 / 1981$ \\
\hline 71 & $\begin{array}{c}\text { Medio San Juan Litoral del San } \\
\text { Juan }\end{array}$ & Chagpien Tordo - Dos Brazos palo bru & $N^{\circ} 075$ de $10 / 11 / 1983$ \\
\hline 72 & Rio Sucio & La Raya & $\mathrm{N}^{\circ} 38$ de $03 / 08 / 1992$ \\
\hline 73 & Bojaya & Rio Napipi & $N^{\circ} 063$ de $10 / 01 / 1986$ \\
\hline 74 & Rio Sucio & Rio Quiparado & $\mathrm{N}^{\circ} 061$ de $21 / 09 / 1981$ \\
\hline 75 & Rio Sucio & Perancho & $\mathrm{N}^{\circ} 037$ de 03/08/1992 \\
\hline 76 & Medio Atrato & Rio Bebará & $\mathrm{N}^{\circ} 038$ de $30 / 11 / 1998$ \\
\hline
\end{tabular}




\begin{tabular}{|c|c|c|c|}
\hline Municipio & Municipio & Nombre del Resgurado & Resolución de constitución \\
\hline 77 & Litoral del San Juan & Santa Marta de Pangala & $N^{\circ} 015$ de $18 / 02 / 1987$ \\
\hline 78 & Nuqui & Rio Pangui & $N^{\circ} 088$ de $27 / 07 / 1982$ \\
\hline 79 & Acandi & Pescadito & $\mathrm{N}^{\circ} 007$ de $20 / 02 / 2001$ \\
\hline 80 & Juradó & Katio & $N^{\circ} 102$ de $27 / 07 / 1982$ \\
\hline 81 & Quibdó & El 20 la Playa Alta- Tutunendo el 90 & $N^{\circ} 083$ de $01 / 07 / 1982$ \\
\hline 82 & Carmen del Atrato & LA Cristalina & $N^{\circ} 014$ de $29 / 06 / 2000$ \\
\hline 83 & Quibdó & Neguá - Necorá & $N^{\circ} 022$ de $24 / 04 / 1985$ \\
\hline 84 & Quibdó & Mungarado & $\mathrm{N}^{\circ} 037$ de $31 / 05 / 1999$ \\
\hline 85 & Quibdó & Rio Munguido & $N^{\circ} 017$ de $23 / 06 / 2000$ \\
\hline 86 & Litoral del San Juan & Unión Chocó San Cristobal & $N^{\circ} 036$ de 22/05/1984 \\
\hline 87 & Medio Baudó & Sirena Berrecuy & $N^{\circ} 029$ de $24 / 09 / 2001$ \\
\hline 88 & Alto Baudó & Dearade Biakirude & $\mathrm{N}^{\circ} 013$ de $29 / 06 / 200$ \\
\hline 89 & Quibdó (Medio Atrato) & Rio Amé & $N^{\circ} 079$ de $17 / 12 / 1990$ \\
\hline 90 & Medio Baudó & Patio Bonito & $N^{\circ} 016$ de $10 / 04 / 2003$ \\
\hline 91 & Alto y Medio Baudó & Catrú- Dubasa Ancoso & $\mathrm{N}^{\circ} 014$ de $21 / 04 / 1982$ \\
\hline 92 & Alto Baudó & Dominico Londoño y Apartadó & $N^{\circ} 073$ de $19 / 11 / 1990$ \\
\hline 93 & Alto Baudó & Pto. Alegre La Divisa Rio Nauca & $\mathrm{N}^{\circ} 42$ de $21 / 07 / 1988$ \\
\hline 94 & Alto Baudó & Puerto Libia Tripicay & $N^{\circ} 41$ de $30 / 11 / 1998$ \\
\hline 95 & Bahía Solano & Villa Nueva Juná & $\mathrm{N}^{\circ} 001$ de $27 / 02 / 2002$ \\
\hline 96 & Carmen del Atrato & La Puria & $\mathrm{N}^{\circ} 056$ de $23 / 12 / 1998$ \\
\hline 97 & Unguía & Tanela & $N^{\circ} 101$ de $27 / 07 / 1982$ \\
\hline 98 & Carmen del Atrato & Sabaleta & $\mathrm{N}^{\circ} 01$ de 14/04/1997 \\
\hline 99 & Bojayá & Puerto Antioquia & $N^{\circ} 42$ de $30 / 11 / 1998$ \\
\hline 100 & Rio Sucio & Peranchito & $\mathrm{N}^{\circ} 39$ de 03/08/1992 \\
\hline 101 & Bajo Baudó & Puerto Chichiliano & $\mathrm{N}^{\circ} 017$ de $10 / 04 / 2003$ \\
\hline 102 & Quibdó (Rio Quito) & La lomita & $\mathrm{N}^{\circ} 055$ de $23 / 12 / 1998$ \\
\hline 103 & Tadó & Bochoromá - Bochoromacito & $\mathrm{N}^{\circ} 071$ de $29 / 04 / 1988$ \\
\hline 104 & Vigía del Fuerte (Antioquia) & Jengadó Apartadó & $\mathrm{N}^{\circ} 015$ de $25 / 03 / 1992$ \\
\hline 105 & Vigía del Fuerte (Antioquia) & El Salado & $\mathrm{N}^{\circ} 022$ de $26 / 03 / 1990$ \\
\hline 106 & Rio Sucio & Peña Blanca Rio Truandó & $\mathrm{N}^{\circ} 040$ de $03 / 08 / 1982$ \\
\hline 107 & Rio Sucio & Yarumal Barranco & $N^{\circ} 042$ de 09/08/1992 \\
\hline 108 & Tadó & Peñas del olvido & $N^{\circ} 004$ de $11 / 05 / 1998$ \\
\hline 109 & Novita y Condoto & Alto Bonito Vira Vira & $N^{\circ} 016$ de $10 / 12 / 2002$ \\
\hline 110 & Sipi & Sanandocito & $N^{\circ} 008$ de $20 / 02 / 2001$ \\
\hline 111 & Tadó & TArena & $N^{\circ} 073$ de $29 / 08 / 1988$ \\
\hline 112 & Sipi El Dovio (Valle del Cauca) & Rio Garrapatas & $N^{\circ} 043$ de $11 / 06 / 1987$ \\
\hline 113 & Juradó & Nussi Parrú & $N^{\circ} 035$ de $03 / 10 / 2000$ \\
\hline 114 & San Jose del Palmar & Copé del Rio Ingará & $N^{\circ} 006$ de $24 / 05 / 1996$ \\
\hline 115 & Novita & Sabaletera Monteria y San Onofre & $N^{\circ} 019$ de $10 / 04 / 2003$ \\
\hline 116 & Unguia & Arquia & $N^{\circ} 100$ de $27 / 07 / 1982$ \\
\hline
\end{tabular}




\begin{tabular}{cccc}
\hline Municipio & Municipio & Nombre del Resgurado & Resolución de constitución \\
\hline 117 & Carmen Del Atrato & El Feria & $\mathrm{N}^{\circ} 224$ de $26 / 10 / 2010$ \\
118 & BOJAYA & Gegenadó & $\mathrm{N}^{\circ} 237$ de $23 / 12 / 2010$ \\
119 & Tadó & El Silencio & $\mathrm{N}^{\circ} 238$ de $23 / 12 / 2010$ \\
120 & Unguía & Embera Dobida Dogibi & $\mathrm{N}^{\circ} 303$ de $17 / 04 / 2013$ \\
121 & Rio Quito & Rios Pato y Jengado & $\mathrm{N}^{\circ} 39$ de $21 / 07 / 1988$
\end{tabular}

